\title{
Anacardic acid induces cell apoptosis of prostatic cancer through autophagy by ER stress/DAPK3/Akt signaling pathway
}

\author{
JING TAN, XIANZHEN JIANG, GUANGMING YIN, LEYE HE, \\ JIANYE LIU, ZHI LONG, ZHIQIANG JIANG and KUN YAO
}

\begin{abstract}
Department of Urology, The Third Xiangya Hospital of Central South University; Institute of Prostate Disease, Central South University, Changsha, Hunan 410013, P.R. China
\end{abstract}

Received February 4, 2017; Accepted July 3, 2017

DOI: $10.3892 / o r .2017 .5841$

\begin{abstract}
Anacardic acid, which is commonly seen in plants of Anacardiaceae, is an important composition of cashew, ginkgo leaf and fruit, and it has been suggested in previous research to show antitumor activity. The main aim of the present study was to evaluate the anticancer effects of anacardic acid on cell apoptosis of prostatic cancer and molecular mechanisms of this phenomenon. In this study we found that anacardic acid inhibited cell proliferation, induced apoptosis and caspase3/9 activities and Bax protein expression of prostatic cancer. Anacardic acid induced the ER stress inducing factors (BiP, CHOP, p-eIF2 $\alpha$ ), autophagy, LC3, Beclin-1, Atg 7 and DAPK3 protein expression, and suppressed p-Akt and p-mTOR protein expression of prostatic cancer. Si-CHOP was used to inhibit ER stress in prostatic cancer by anacardic acid, which showed that the cell proliferation was increased, apoptosis, and caspase-3/9 activities and Bax protein expression was suppressed, autophagy, LC3, Beclin-1, Atg 7 and DAPK3 protein expression was reduced, and p-Akt and p-mTOR protein expression was promoted. DAPK 3 inhibited p-Akt and p-mTOR protein expression, enhanced the anticancer effects of anacardic acid on prostatic cancer through autophagy. For the first time, the present study showed that anacardic acid induces cell apoptosis of prostatic cancer through autophagy by ER stress/DAPK3/Akt signaling pathway.
\end{abstract}

\section{Introduction}

The morbidity of prostate cancer in the world is $25.3 / 100,000$, which ranks in the second place among all malignant tumors in male (1). In addition, its morbidity is associated with distinct geographical and racial differences, with countries such as America, Canada, Australia/New Zealand, Northern

Correspondence to: Dr Kun Yao, Department of Urology, The Third Xiangya Hospital of Central South University; Institute of Prostate Disease, Central South University, Changsha, Hunan 410013, P.R. China

E-mail: yaokun52@163.com

Key words: anacardic acid, prostatic cancer, autophagy, ER stress
Europe and Western Europe having higher morbidity (1). The morbidity of prostate cancer in America has surpassed that of lung cancer, making it the top tumor threatening male health. It is estimated by the American Cancer Society that approximately 217,730 new prostate cancer cases occurred in America in 2010, and 32,050 of them die of such disease (2). Prostate cancer is the most common cancer in male in European Union, which accounts for $18.1 \%$ of all new cases. Approximately 2.6 million out of the new prostate cancer cases are confirmed every year. Prostate cancer takes up $11 \%$ of all male cancers and accounts for $9 \%$ of all male cancer deaths (3).

Autophagy is an evolutionally conserved process in eukaryotic cell that is regulated by genes. During such dynamic process, regulating degradation of intracellular proteins and organelles contributes to the formation of intracellular autophagosome with double-layer membrane structure, so that cytoplasm, proteins and organelles can be partly degraded for recycling (4). The formation of autophagosome is a multi-step process involving multiple autophagosome-related proteins and 2 ubiquitin-like covalence systems (5). Such process can induce fusion of autophagosome with lysosome to form the autophagic lysosome; and lysosomal hydrolase will degrade the contents that it swallows (5). Autophagy is effective in treating prostate cancer, because it can enhance sensitivity of tumor cells to various therapies, including DNA damaging agent, anti-hormone therapy (such as aniline) and radiotherapy (6).

Endoplasmic reticulum (ER) is a kind of membrane-bound organelle in eukaryotic cells, which is mainly responsible for the correct folding and post-translational modification of membrane proteins and secretory proteins (7). In addition, ER also plays an important role in biosynthesis of lipids, energy metabolism, intracellular $\mathrm{Ca}^{2+}$ homeostasis and redox equilibrium. Protein folding function in ER is extremely sensitive to extracellular and intracellular stimulations, including ischemia reperfusion, inflammation, glycosylation and $\mathrm{Ca}^{2+}$ disequilibrium (8). Aggregation of misfolded or unfolded proteins in ER lumen will induce endoplasmic reticulum stress (ER stress), thus, activating unfolded protein response (9).

Death-associated protein kinase-3 (DAPk3) is a kind of $\mathrm{Ca}^{2+} / \mathrm{CaM}$-regulating serine/threonine protein kinase discovered by Israel scientist Adi Kimchi by means of gene knockout in 1995. At first, DAPk3 was discovered as a canonical tumor suppressor gene (10). In recent years, increasing studies have 

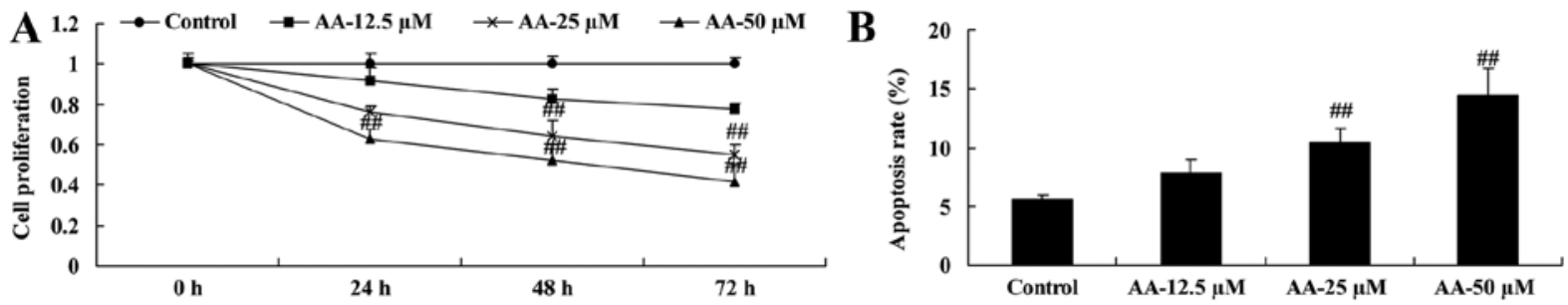

C

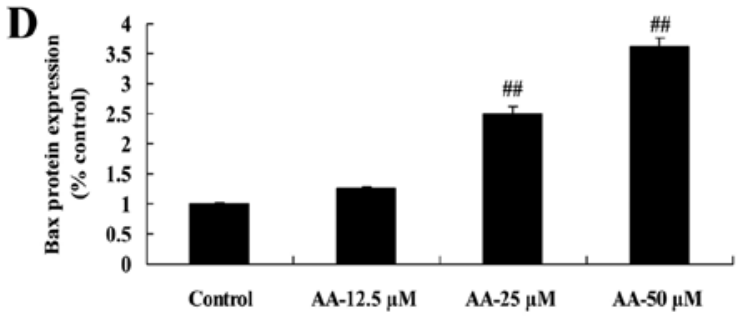

GAPDH
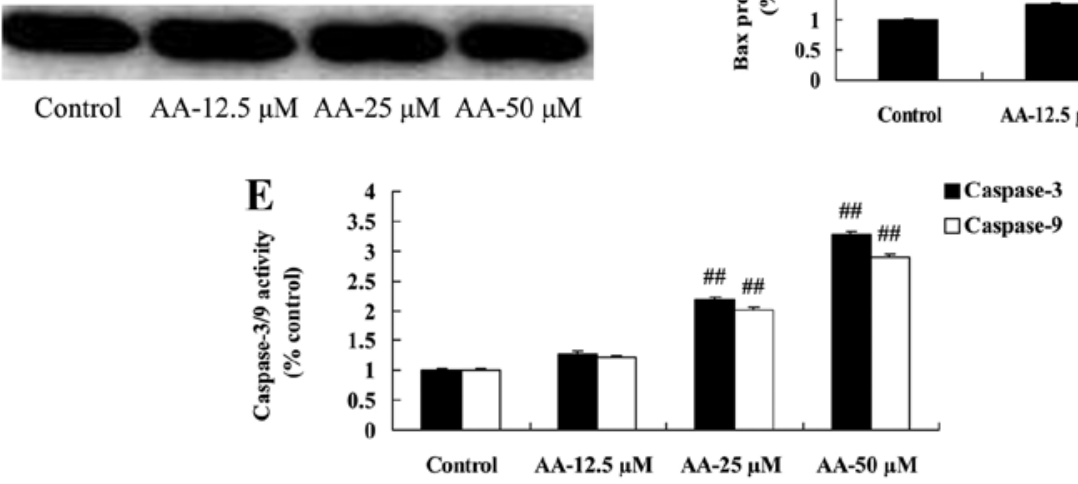

Figure 1. The effects of anacardic acid on cell proliferation and apoptosis of prostatic cancer cells. The effects of anacardic acid on cell proliferation (A), apoptosis (B), Bax protein expression using western blot analysis and statistical analysis (C and D) and caspase-3/9 activities (E) of prostatic cancer cell. ${ }^{\# \#} \mathrm{P}<0.01$ vs. control group.

verified that DAPk3 is involved in multiple cellular functions. In addition, it participates in multiple signal pathways to regulate apoptosis, autophagy, caspase-dependent cell death, adhesion and migration (11). Therefore, it not only plays a certain role in inflammatory response, but also exerts antitumor functions inhibiting metastasis (11).

PI3K/Akt pathway is one of the important intracellular signal transduction pathways, which exerts extremely important biological effects in multiple physiological functions, such as cell growth, proliferation, apoptosis, angiogenesis and autophagy, as well as development and protection of the nervous system (12). Disorder of such pathway will result in multiple diseases, such as cancer genesis and progression, nervous system disease, autoimmune disease and hematopoietic system disease (13).

Traditional Chinese herbal medicines are verified to be extremely effective through thousands of years of practice, but compositions and mechanisms of action of numerous Chinese herbal medicines remain unclear (14). Therefore, Chinese herbal medicine monomer with safe and wide source as well as definite action is the first choice in drug screening (15). Bark of plants of Anacardiaceae is frequently used in treating gastric ulcer, gastritis and gastric cancer in Mexico (16). Anacardic acid is an important antitumor bioactive substance purified from Amphipterygium adstringens, the bark of which is used traditionally to treat gastric ulcer, gastritis and gastric cancer (17). Recent research has indicated that anacardic acid possesses the effects of inhibiting proliferation of multiple tumor cells (such as lung cancer, liver cancer, multiple myeloma and prostate cancer) and inducing apoptosis (18). In the present study, we investigated the anticancer effects of anacardic acid on cell apoptosis of prostatic cancer and molecular mechanisms of this phenomenon.

\section{Materials and methods}

Cell lines and cell culture. Human prostate carcinoma cell line LNCaP cells were obtained from the American Type Cell Culture (ATCC; Manassas, VA, USA) and cultured in Dulbecco's modified Eagle's medium (DMEM; HyClone Laboratories, Inc., Logan, UT, USA), $10 \%$ fetal bovine serum (FBS; HyClone Laboratories) at $37^{\circ} \mathrm{C}$ and $5 \% \mathrm{CO}_{2}$.

Proliferation assay. LNCaP cells were treated with $12.5,25$ and $50 \mu \mathrm{M}$ of anacardic acid for 24,48 and $72 \mathrm{~h}$. 3-(4,5-dimethylthiazol-2-yl)-2,5-diphenyl-2H-tetrazolium bro mide (MTT; Sigma-Aldrich, St. Louis, MO, USA) was added into cells and incubated for $4 \mathrm{~h}$ at $37^{\circ} \mathrm{C}$. Old medium was removed and dimethyl sulfoxide (DMSO) was added for solution at $37^{\circ} \mathrm{C}$ for $20 \mathrm{~min}$. The intensity was measured using an ELISA reader (Molecular Devices, Sunnyvale, CA, USA) at $490 \mathrm{~nm}$.

Apoptosis assay. LNCaP cells were treated with 12.5, 25 and $50 \mu \mathrm{M}$ of anacardic acid for $48 \mathrm{~h}$. LNCaP cells were stained with Annexin V/FITC (Becton-Dickinson, San Jose, CA, USA) and prodium iodide (PI; Becton-Dickinson) at darkness for $30 \mathrm{~min}$. Apoptosis was quantitatively estimated on a FACScan flow cytometry (Becton-Dickinson). 


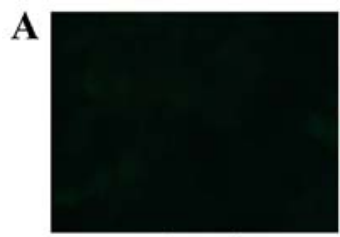

Control

B

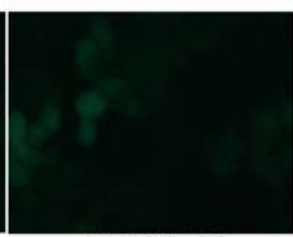

AA-12.5 $\mu \mathrm{M}$

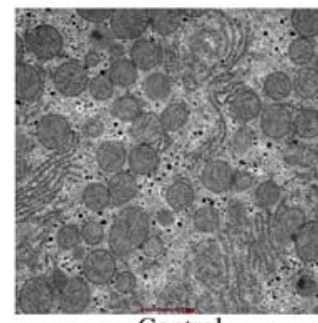

Control

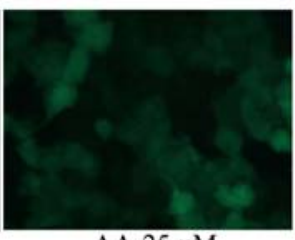

$\mathrm{AA}-25 \mu \mathrm{M}$

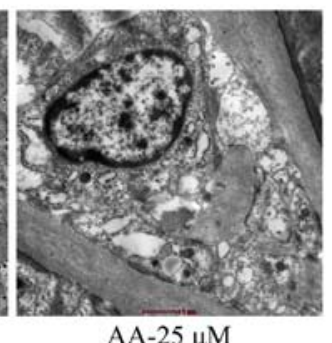

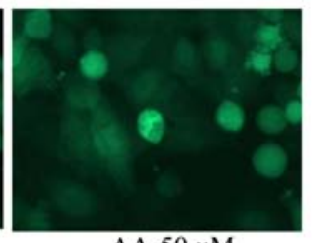

$\mathrm{AA}-50 \mu \mathrm{M}$

Figure 2. The effects of anacardic acid on autophagy of prostatic cancer cells. The effects of anacardic acid on LC3 protein expression (A) and electron microscope (B) of prostatic cancer cell. $\mathrm{P}<0.01$ vs. control group.

$\mathbf{A}_{\text {LC3 }}$
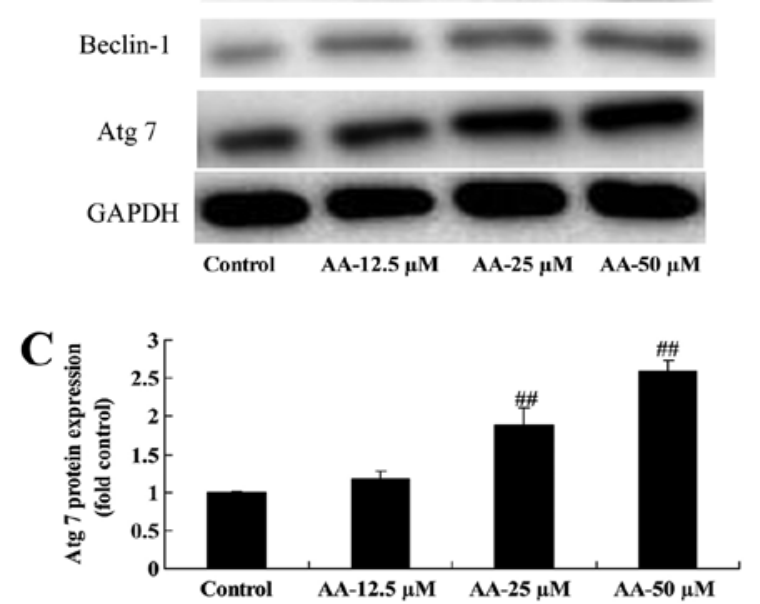

B
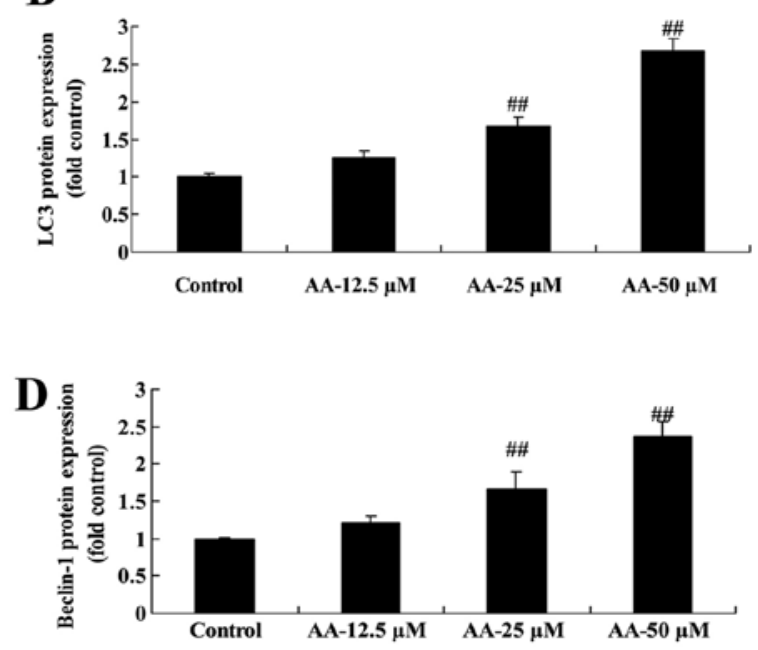

Figure 3. The effects of anacardic acid on autophagy associated protein of prostatic cancer cells. The effects of anacardic acid on LC3, Beclin-1 and Atg 7 protein expression using western blot analysis (A) and statistical analysis for LC3, Beclin-1 and Atg 7 protein expression (B-D) of prostatic cancer cells. ${ }^{\# \#} \mathrm{P}<0.01$ vs. control group.

Western blot analysis. Cells were washed with phosphatebuffered saline (PBS) and split using RIPA assay (Pierce, Rockford, IL, USA). The protein was quantified by a BCA assay (Pierce). Denatured proteins were separated by $8-12 \%$ sodium dodecyl sulfate-polyacrylamide gel electrophoresis (SDS-PAGE) and then transferred to nitrocellulose (Amersham, Bensheim, Germany). Nitrocellulose was blocked with 5\%-BSA in TBST and incubated with Bax, BiP, CHOP, p-eIF2 $\alpha$, LC3, Beclin-1, Atg 7, DAPK3, p-Akt, p-mTOR and GAPDH (Santa Cruz Biotechnology, Santa Cruz, CA, USA) at $4^{\circ} \mathrm{C}$ overnight. Nitrocellulose was washed with TBST, incubated with horseradish peroxidase (HRP)-conjugated appropriate secondary antibodies and reacted with ECL detection reagents (Amersham Bioscience).

Statistical analysis. All data are shown as mean \pm SD. Comparison of multiple groups was made with the one-way ANOVA followed by the Tukey's test or the Newman-Keuls test. Differences with $\mathrm{P}<0.05$ were considered statistically significant.

\section{Results}

The effects of anacardic acid on cell proliferation and apoptosis of prostatic cancer cells. In our initial study, cell proliferation and apoptosis of prostatic cancer cell were determined. As shown in Fig. 1A and B, anacardic acid effectively inhibited cell proliferation and induced apoptosis of prostatic cancer cells. Moreover, anacardic acid significantly induced Bax promoted and caspase-3/9 activities of prostatic cancer cell (Fig. 1C-E).

The effects of anacardic acid on autophagy of prostatic cancer cells. We evaluated the anticancer effects of anacardic acid on autophagy of prostatic cancer cells. As shown in Fig. 2A, anacardic acid caused autophagy and LC3 protein 
A

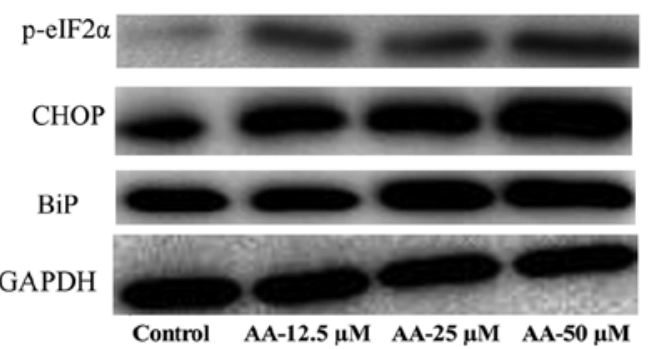

C

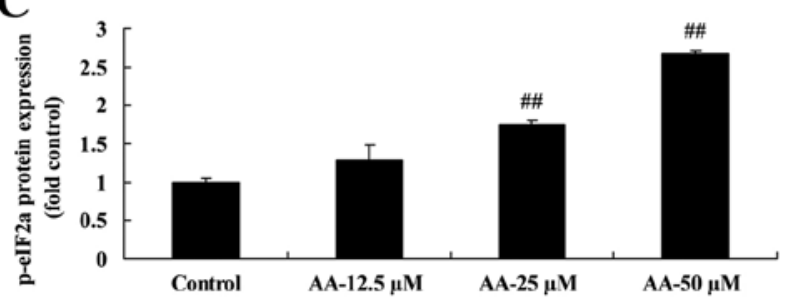

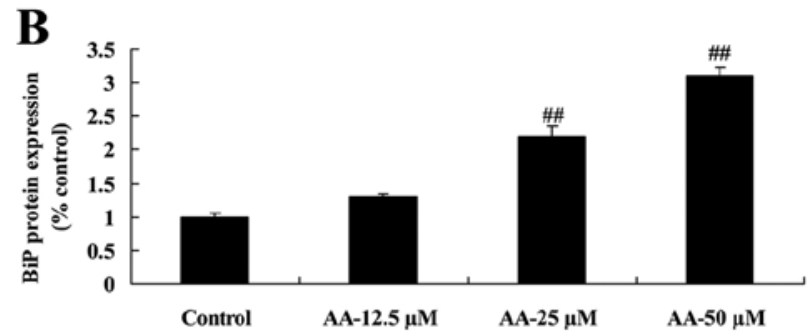

D

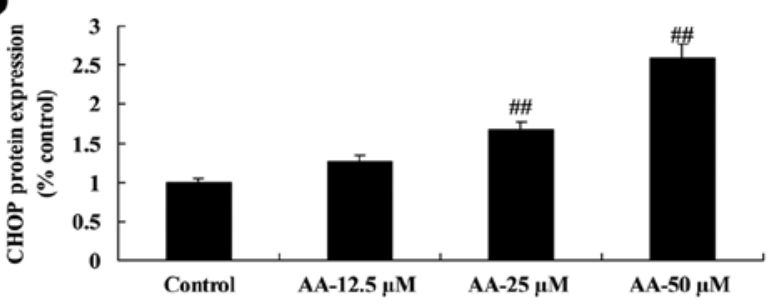

Figure 4. The effects of anacardic acid on ER stress of prostatic cancer cells. The effects of anacardic acid on BiP, CHOP and p-eIF2 $\alpha$ protein expression using western blot analysis (A) and statistical analysis for BiP, CHOP and p-eIF $2 \alpha$ protein expression (B-D) of prostatic cancer cells. ${ }^{\# \#} \mathrm{P}<0.01$ vs. control group.

A
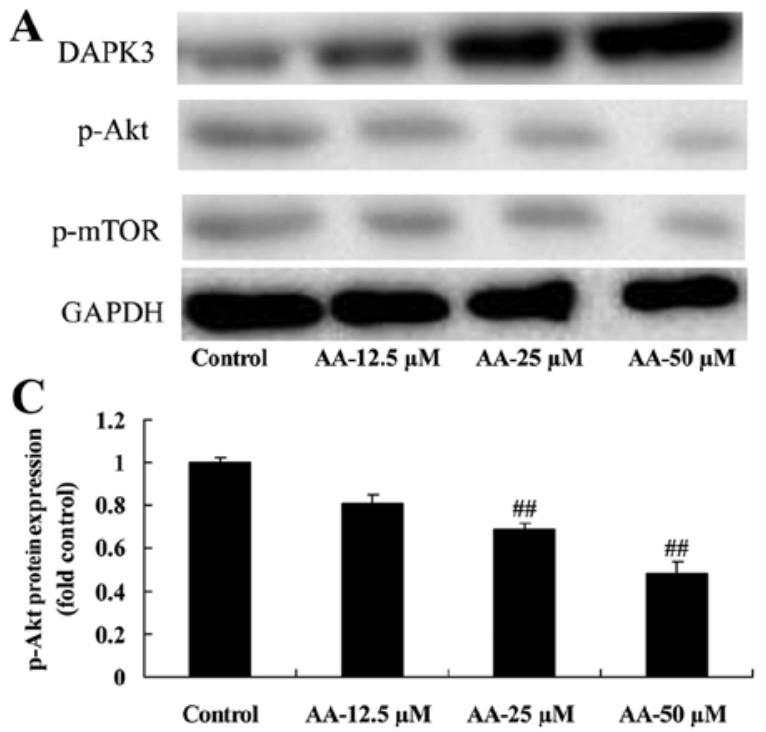

B

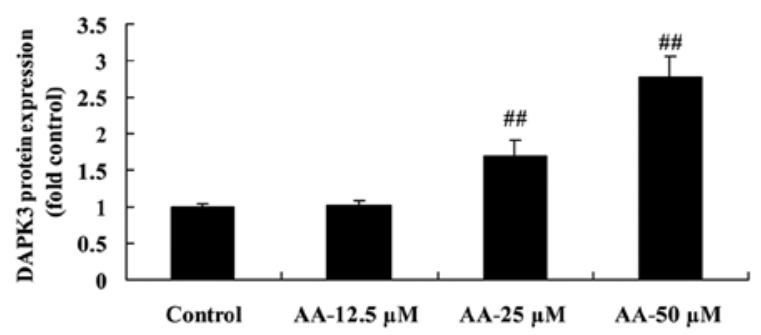

D

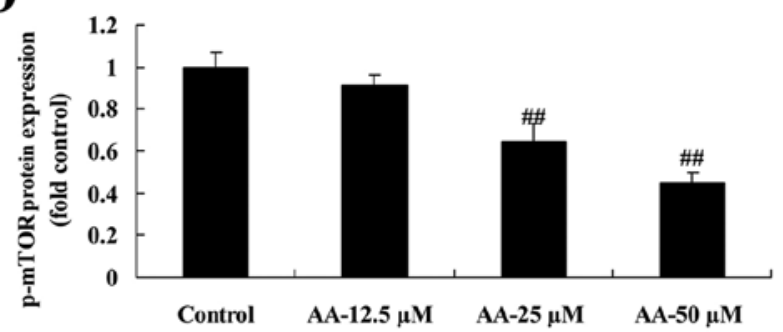

Figure 5. The effects of anacardic acid on DAPK3/Akt signaling of prostatic cancer cells. The effects of anacardic acid on DAPK3, p-Akt and p-mTOR protein expression using western blot analysis (A) and statistical analysis for DAPK3, p-Akt and p-mTOR protein expression (B-D) of prostatic cancer cell. ${ }^{\# \# ~} \mathrm{P}<0.01$ vs. control group.

expression of prostatic cancer cells in a dose-dependent manner. Anacardic acid significantly produced autophagy vesicles of prostatic cancer cells (Fig. 2B).

The effects of anacardic acid on autophagy associated protein of prostatic cancer cells. We determined whether the effects of anacardic acid on autophagy associated with protein of prostatic cancer cells. The results in Fig. 3 revealed that anacardic acid significantly induced LC3, Beclin-1 and Atg 7 protein expression of prostatic cancer cells in a dose-dependent manner.

The effects of anacardic acid on ER stress of prostatic cancer cells. To determine whether ER stress participates in the anticancer effects of anacardic acid on prostatic cancer cell growth, BiP, CHOP and p-eIF2 $\alpha$ protein expression was measured using western blot analysis. The results in Fig. 4 showed that anacardic acid significantly increased ER stress, and induced BiP, CHOP and p-eIF2 $\alpha$ protein expression of prostatic cancer cells in a dose-dependent manner.

The effects of anacardic acid on DAPK3/Akt signaling of prostatic cancer cells. In order to further prove the autophagy induced by anacardic acid in prostatic cancer cells, DAPK3/Akt signaling was measured using western blot analysis. Treatment with anacardic acid significantly induced DAPK 3 and suppressed p-Akt and p-Mtor protein expression in prostatic cancer cells in a dose-dependent manner (Fig. 5).

The inhibition of ER stress in prostatic cancer cell treated by anacardic acid. To investigate whether ER stress participated in apoptosis of prostatic cancer cells treated by anacardic acid, we performed si-CHOP transiently. The induction of BiP, 
A

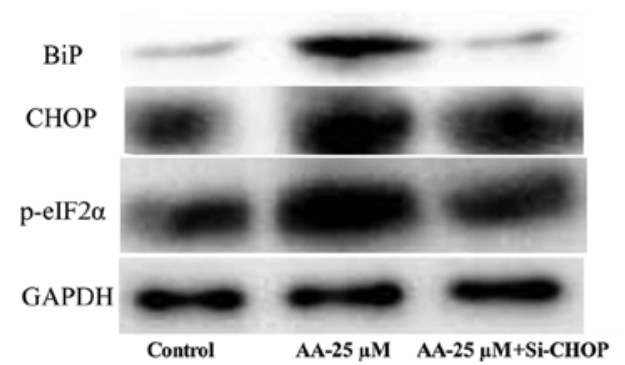

C

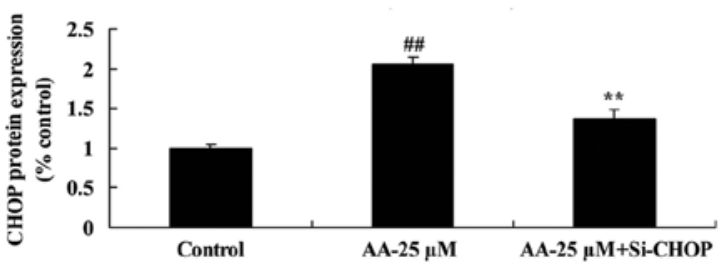

B

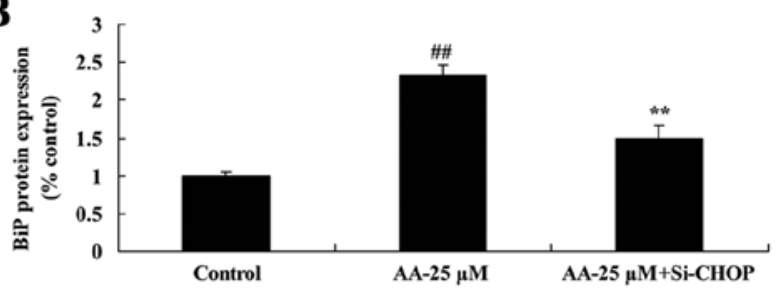

D

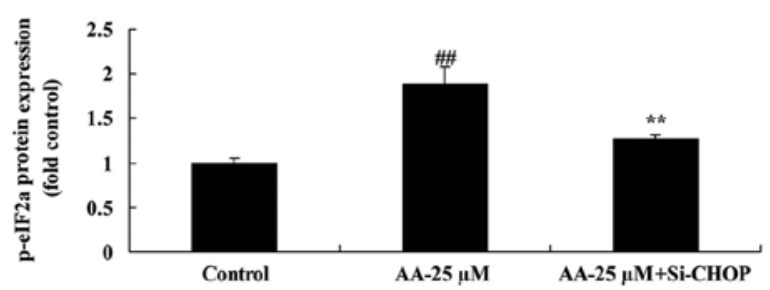

Figure 6. The inhibition of ER stress in prostatic cancer cells treated by anacardic acid. The inhibition of ER stress BiP, CHOP and p-eIF2 $\alpha$ protein expression using western blot analysis (A) and statistical analysis for BiP, CHOP and p-eIF2 $\alpha$ protein expression (B-D) in prostatic cancer cell treated by anacardic acid. ${ }^{\# \#} \mathrm{P}<0.01$ vs. control group, ${ }^{\# \#} \mathrm{P}<0.01$ vs. AA $-25 \mu \mathrm{M}$ group.

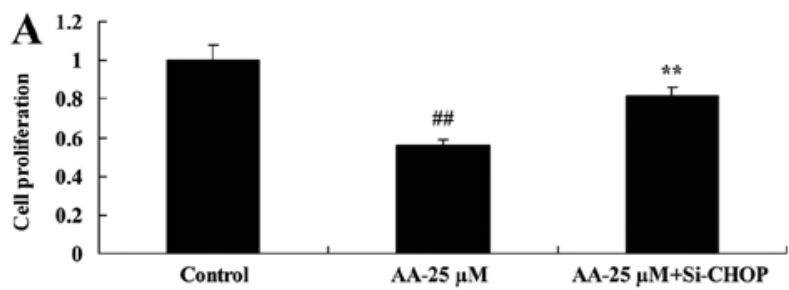

C

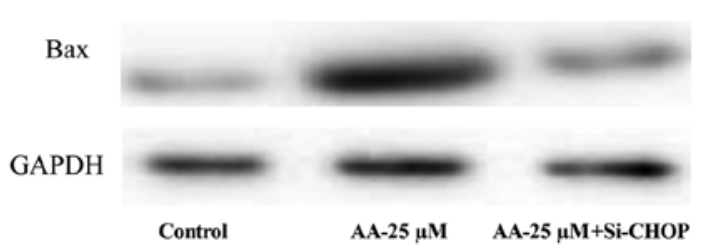

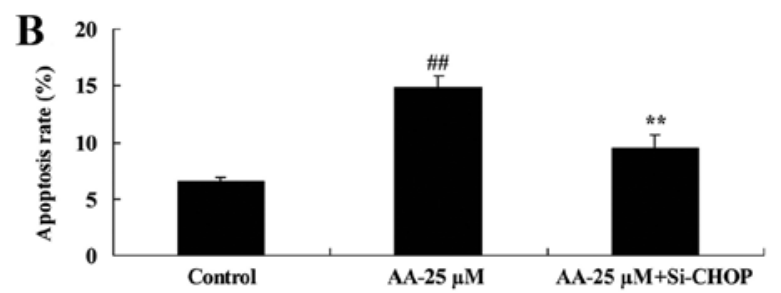

D

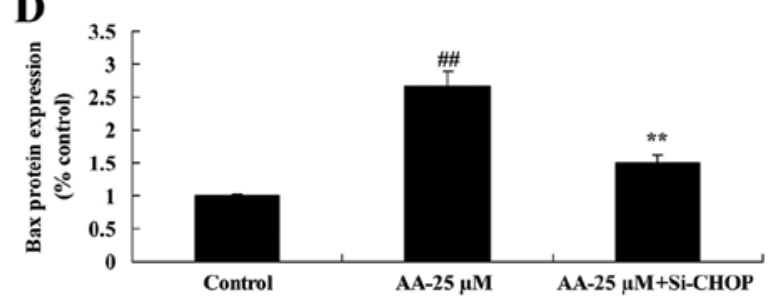

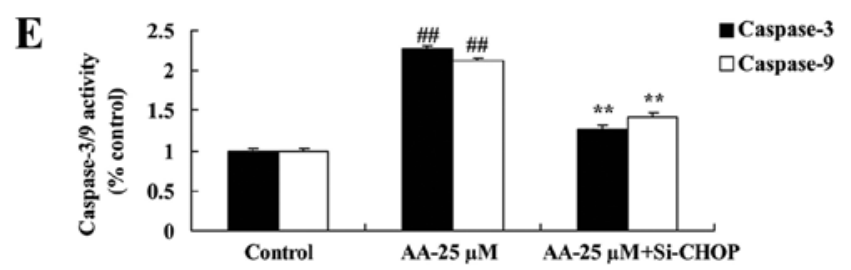

Figure 7. The inhibition of ER stress on cell proliferation and apoptosis of prostatic cancer cells treated by anacardic acid. The inhibition of ER stress on cell proliferation (A), apoptosis (B), Bax protein expression using western blot analysis and statistical analysis (C and D) and caspase-3/9 activities (E) of prostatic cancer cells by anacardic acid. ${ }^{\# \#} \mathrm{P}<0.01$ vs. control group, ${ }^{* *} \mathrm{P}<0.01$ vs. AA- $25 \mu \mathrm{M}$ group.

CHOP and p-eIF2 $\alpha$ protein expression by anacardic acid was significantly suppressed in prostatic cancer cells (Fig. 6).

The inhibition of ER stress on cell proliferation and apoptosis of prostatic cancer cells treated by anacardic acid. We tested the inhibition of ER stress on cell proliferation and apoptosis of prostatic cancer cells treated by anacardic acid. The result indicated that the inhibition of ER stress significantly reversed the anticancer effects of anacardic acid on the cell proliferation inhibition and induction of apoptosis, Bax and caspase-3/9 activities of prostatic cancer cells (Fig. 7).
Inhibition of ER stress on autophagy of prostatic cancer cells treated by anacardic acid. Fig. 8 shows that the inhibition of ER stress significantly inhibited autophagy and LC3 protein expression of prostatic cancer cells.

Inhibition of ER stress on autophagy associated protein of prostatic cancer cells treated by anacardic acid. Next, to investigate crosstalk between ER stress and autophagy in prostatic cancer cells treated by anacardic acid, we examined LC3, Beclin-1 and Atg 7 protein expression. Notably, the inhibition of ER stress significantly suppressed the increase of 


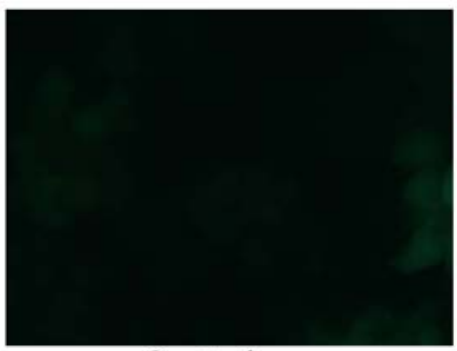

Control

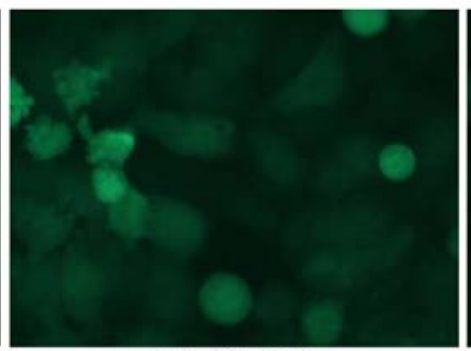

$\mathrm{AA}-25 \mu \mathrm{M}$

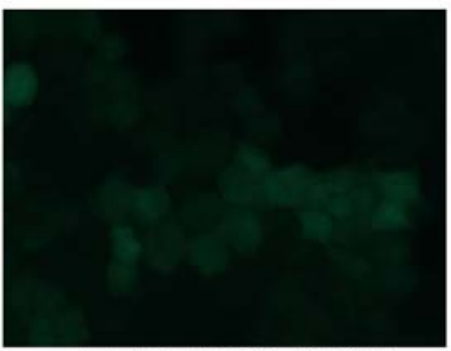

AA- $25 \mu \mathrm{M}+\mathrm{Si}-\mathrm{CHOP}$

Figure 8. The inhibition of ER stress on autophagy of prostatic cancer cell treated by anacardic acid.

A

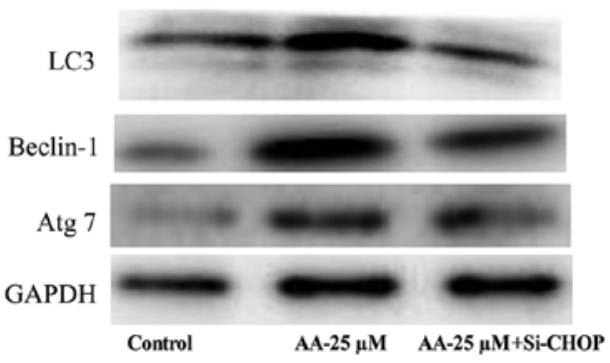

C

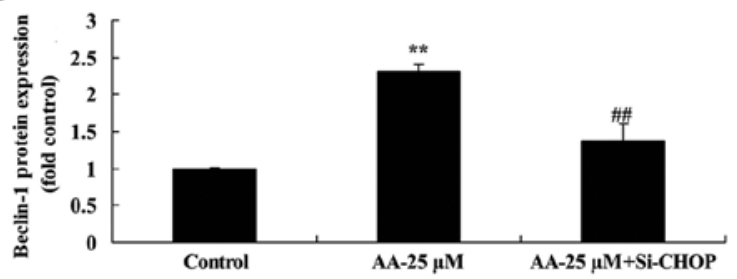

B

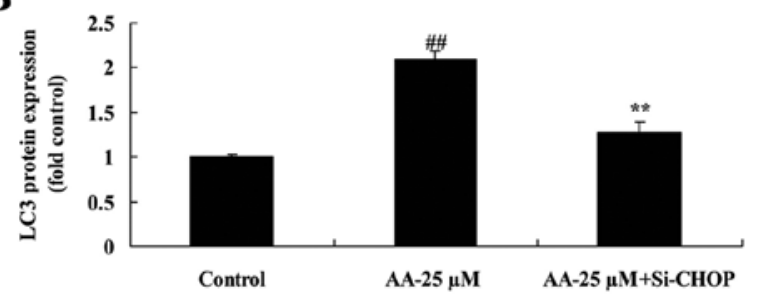

D

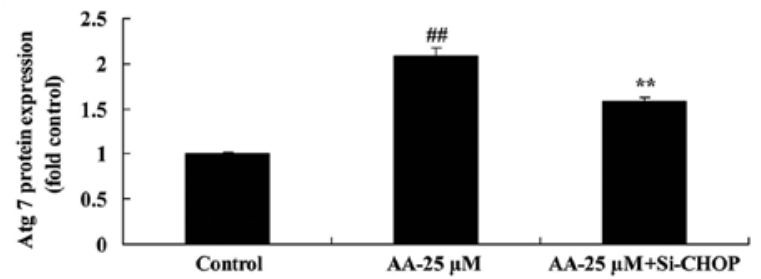

Figure 9. Inhibition of ER stress on autophagy associated protein of prostatic cancer cells treated by anacardic acid. Inhibition of ER stress on LC3, Beclin-1 and Atg 7 protein expression using western blot analysis (A) and statistical analysis for LC3, Beclin-1 and Atg 7 protein expression (B-D) of prostatic cancer cells treated by anacardic acid. ${ }^{\# \#} \mathrm{P}<0.01$ vs. control group, ${ }^{* *} \mathrm{P}<0.01$ vs. AA- $25 \mu \mathrm{M}$ group.

A
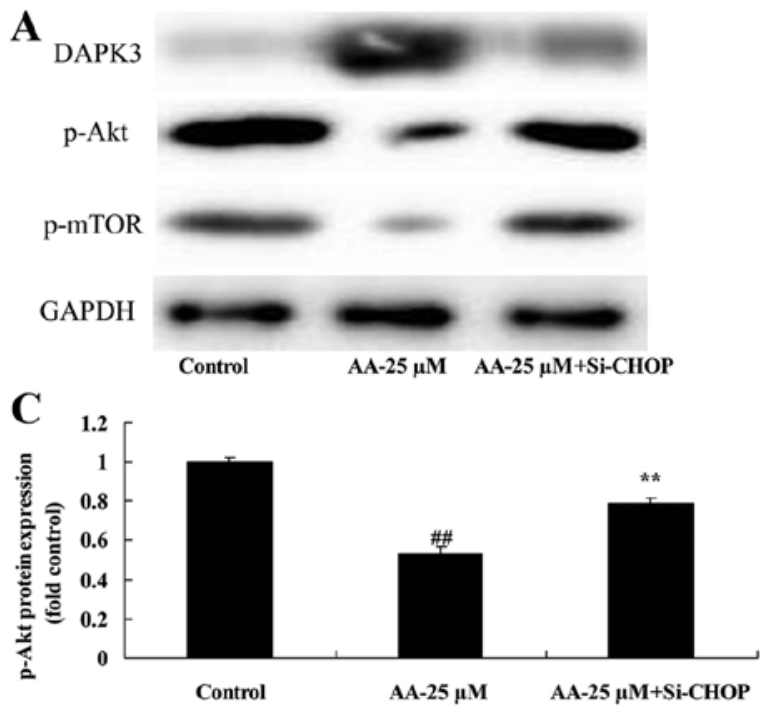

B

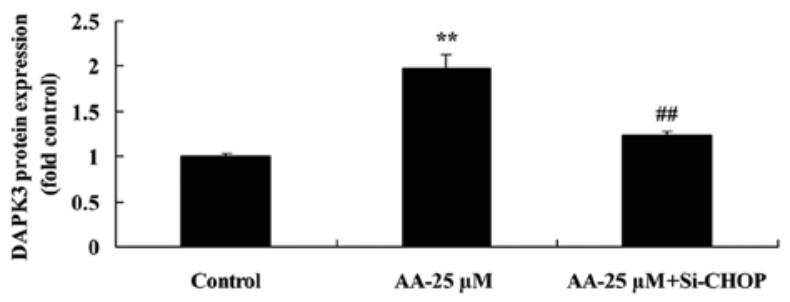

D

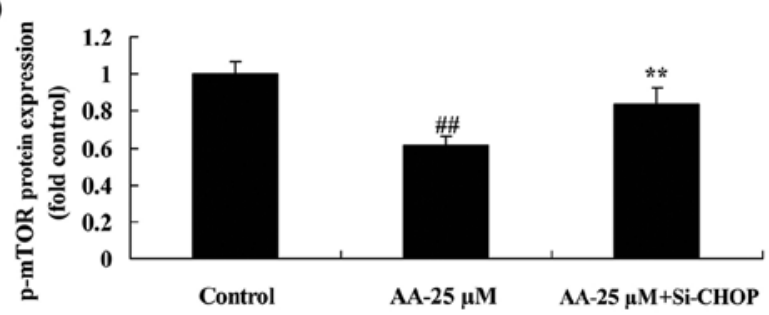

Figure 10. The inhibition of ER stress on DAPK3/Akt signaling of prostatic cancer cell treated by anacardic acid. The inhibition of ER stress on DAPK3, p-Akt and p-mTOR protein expression using western blot analysis (A) and statistical analysis for DAPK3, p-Akt and p-mTOR protein expression (B-D) of prostatic cancer cells treated by anacardic acid. ${ }^{\# \#} \mathrm{P}<0.01$ vs. control group, ${ }^{* *} \mathrm{P}<0.01$ vs. AA- $25 \mu \mathrm{M}$ group.

LC3, Beclin-1 and Atg 7 protein expression of prostatic cancer cells treated by anacardic acid (Fig. 9).
The inhibition of ER stress on DAPK3/Akt signaling of prostatic cancer cell treated by anacardic acid. We further confirmed 
A

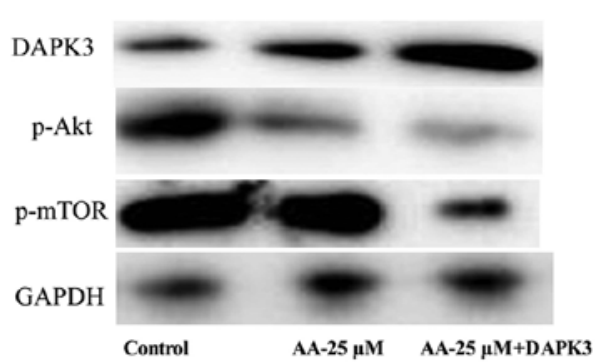

C

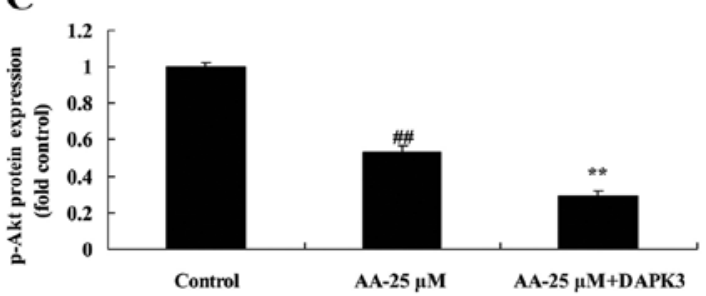

B

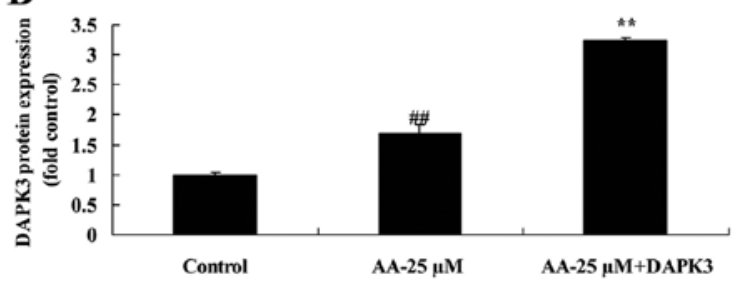

D

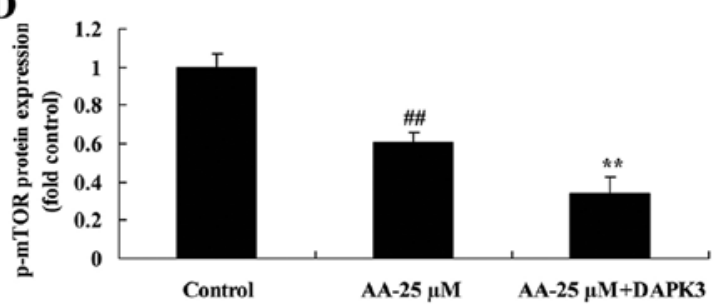

Figure 11. The promotion of DAPK3 on DAPK3/Akt signaling of prostatic cancer cells treated by anacardic acid. The promotion of DAPK3 on DAPK3, p-Akt and p-mTOR protein expression using western blot analysis (A) and statistical analysis for DAPK3, p-Akt and p-mTOR protein expression (B-D) of prostatic cancer cells treated by anacardic acid. ${ }^{\# \#} \mathrm{P}<0.01$ vs. control group, ${ }^{* *} \mathrm{P}<0.01$ vs. AA-25 $\mu \mathrm{M}$ group.

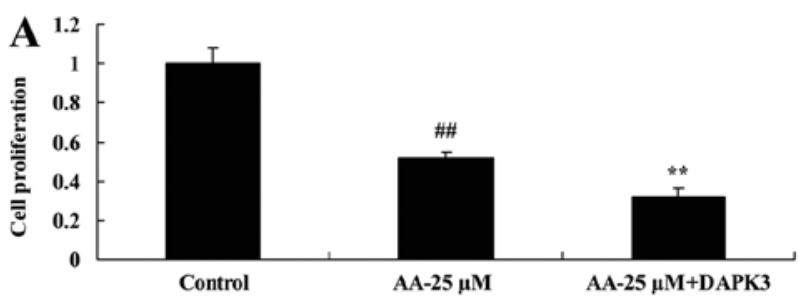

C

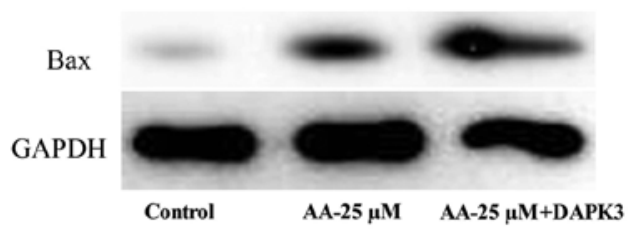

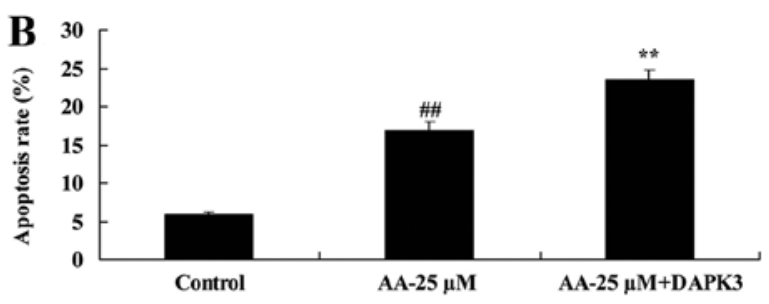

D

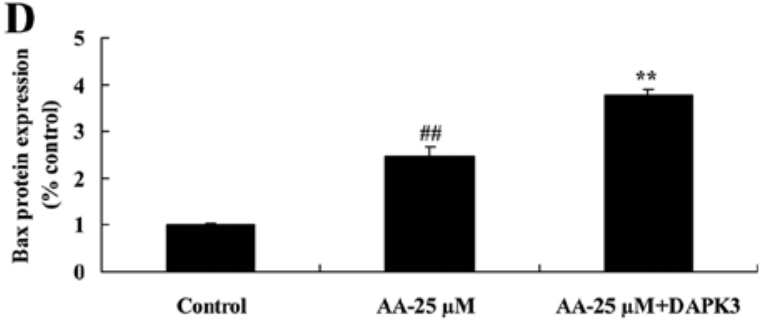

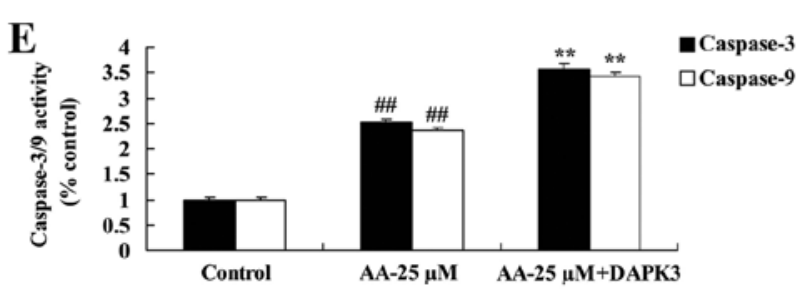

Figure 12. The promotion of DAPK3 on cell proliferation and apoptosis of prostatic cancer cells treated by anacardic acid. The promotion of DAPK3 on cell proliferation (A), apoptosis (B), Bax protein expression using western blot analysis and statistical analysis (C and D) and caspase-3/9 activities (E) of prostatic cancer cells by anacardic acid. ${ }^{\# \#} \mathrm{P}<0.01$ vs. control group, ${ }^{* *} \mathrm{P}<0.01$ vs. AA-25 $\mu \mathrm{M}$ group.

that the inhibition of ER stress affects on DAPK3/Akt signaling of prostatic cancer cell treated by anacardic acid. As shown in Fig. 10, the inhibition of ER stress significantly suppressed DAPK3 protein expression, and induced p-Akt and p-mTOR protein expression of prostatic cancer cells treated by anacardic acid.

The promotion of DAPK3 on DAPK3/Akt signaling of prostatic cancer cell treated by anacardic acid. We next analyzed the role of DAPK3 in DAPK3/Akt signaling of prostatic cancer cell treated by anacardic acid. As shown in Fig. 11, DAPK3 protein increased DAPK3 protein expression, and suppressed $\mathrm{p}$-Akt and p-mTOR protein expression of prostatic cancer cells treated by anacardic acid.

The promotion of DAPK3 on cell proliferation and apoptosis of prostatic cancer cell treated by anacardic acid. We confirmed whether the promotion of DAPK3 on cell proliferation and apoptosis of prostatic cancer cell treated by anacardic acid. The inhibition of cell proliferation and 


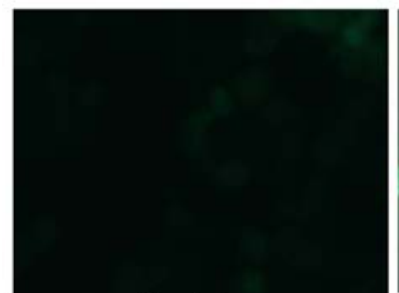

Control

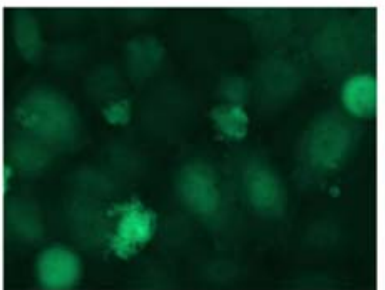

$\mathrm{AA}-25 \mu \mathrm{M}$

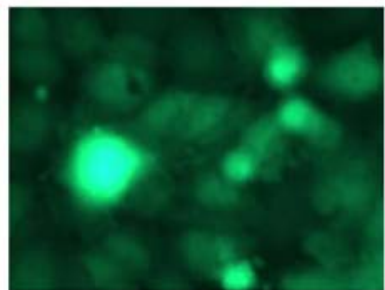

AA- $25 \mu \mathrm{M}+\mathrm{DAPK} 3$

Figure 13. The promotion of DAPK3 on autophagy of prostatic cancer cells treated by anacardic acid.

A

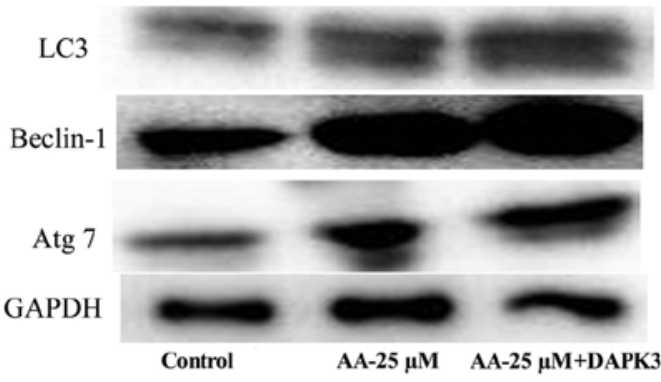

C

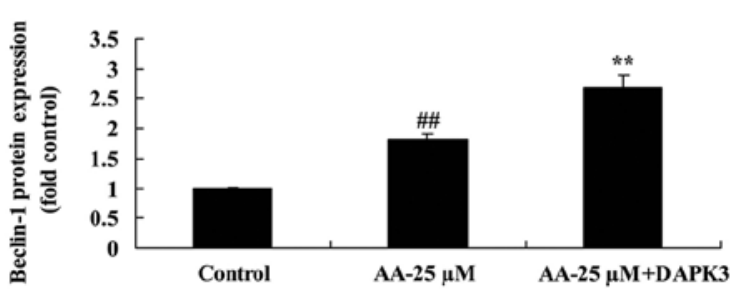

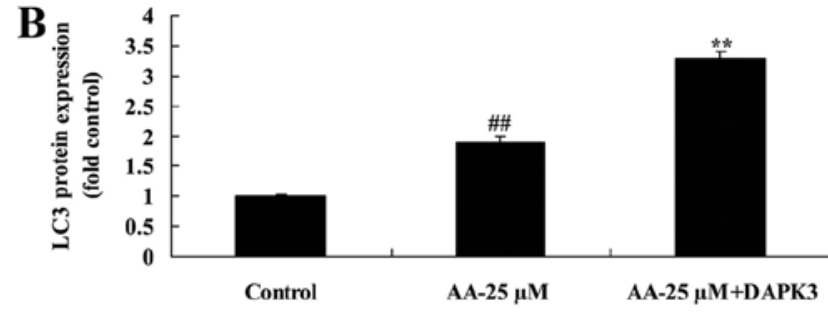

D

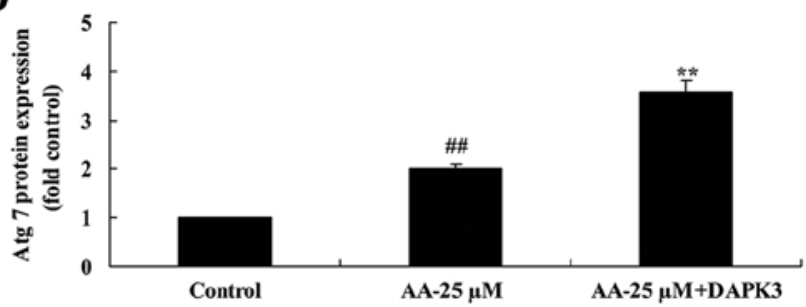

Figure 14. The promotion of DAPK3 on autophagy associated protein of prostatic cancer cells treated by anacardic acid. The promotion of DAPK3 on LC3, Beclin-1 and Atg 7 protein expression using western blot analysis (A) and statistical analysis for LC3, Beclin-1 and Atg 7 protein expression (B-D) of prostatic cancer cells treated by anacardic acid. ${ }^{\# \#} \mathrm{P}<0.01$ vs. control group, ${ }^{* *} \mathrm{P}<0.01$ vs. AA- $25 \mu \mathrm{M}$ group.

promotion of apoptosis of prostatic cancer cell treated by anacardic acid were effectively enhanced by the promotion of DAPK3 (Fig. 12A and B). There were significant increases of Bax protein and caspase-3/9 activities in prostatic cancer cell treated by anacardic acid (Fig. 12C-E).

The promotion of DAPK3 on autophagy of prostatic cancer cell treated by anacardic acid. To confirm the pro-apoptotic function of DAPK3 on autophagy of prostatic cancer cell treated by anacardic acid, we observed autophagy of prostatic cancer cell. The autophagy and LC3 protein expression in prostatic cancer cells treated by anacardic acid were significantly increased by the promotion of DAPK3 (Fig. 13).

The promotion of DAPK3 on autophagy associated protein of prostatic cancer cells treated by anacardic acid. Next, we analyzed autophagy associated protein of prostatic cancer cells treated by anacardic acid + the promotion of DAPK3. DAPK3 overexpressing cells exhibited induction of LC3, Beclin-1 and Atg 7 protein expression of prostatic cancer cells treated by anacardic acid (Fig. 14).

\section{Discussion}

Prostate cancer is a malignant tumor with the highest morbidity in male in America, which is only second to lung cancer and ranks in the second place in malignant tumorrelated mortality, and it takes the third place in Europe (19). In China, the morbidity of prostate cancer is increasing year by year with the universally improved living standard, as well as diagnosis and treatment levels (20). Generally speaking, prostate cancer grows slowly and may be restricted in prostate for years, and many old people are discovered with prostate cancer in autopsy (2). In the present study, we found that anacardic acid inhibited cell proliferation, induced apoptosis, and caspase-3/9 activities and Bax protein expression of prostatic cancer. Yao et al (21) reported that anacardic acid sensitizes radiation therapy-prostate cancer cells by regulating H2AX expression (21).

Autophagy is a genetic programming and evolutionally conserved process, which degrades the excess, harmful or aging proteins and organelles, controls over important cellular components, and plays an important role in normal organism (22). It has been discovered that autophagy is closely related to tumors, which plays an important role in tumor genesis and development (23). In prostate cancer cells, autophagy plays an important role in promoting progression and metastasis of prostate cancer. Besides, it can protect prostate cancer cells from external environment changes, and develops resistance to agents in treatment of prostate cancer (24). Our data showed that anacardic acid induced ER stress prostatic cancer. Moreover, anacardic acid induced 


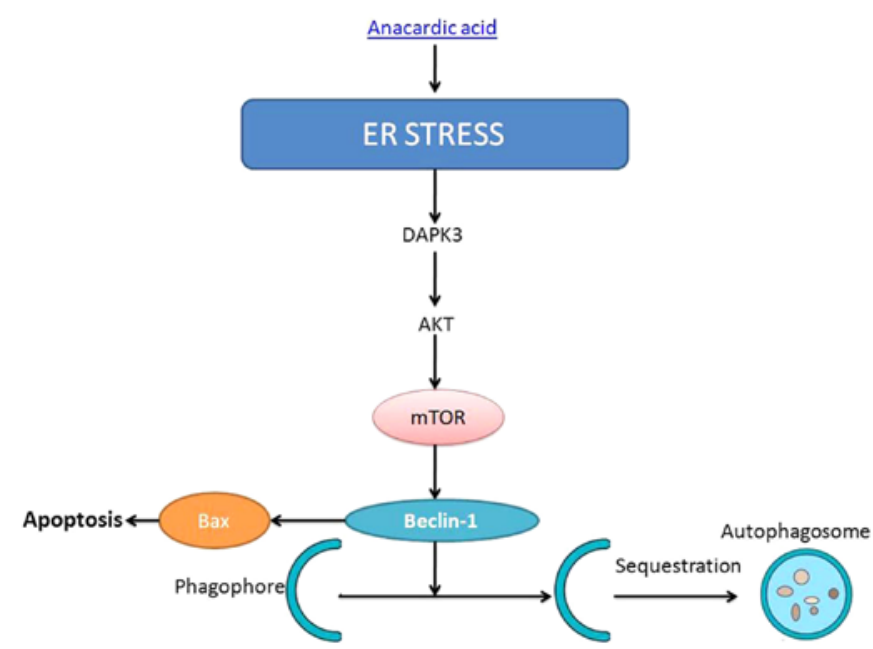

Figure 15. Anacardic acid induces cell apoptosis of prostatic cancer through autophagy by ER stress/DAPK3/Akt signaling pathway.

autophagy and ER stress of prostatic cancer. Seong et al (18) showed that anacardic acid induces ER stress and autophagy of human lung carcinoma A549 cells.

At the early stage of ER stress, protein synthesis within ER will be reduced, and related genes regulating protein translation and correct folding will be activated, which contributes to maintaining the normal physiological functions of cells, thus, promoting cell survival (25). However, a large amount of misfolded or unfolded proteins will be accumulated in ER in the case of excessive ER duration or UPR function impairment, at this moment, pro-apoptosis signal will be activated (26). At present, it is indicated in research that ER dysfunction of myocardial cells and pancreatic cells may be the major pathogenesis leading to cardio-cerebral tissue ischemic blocking and diabetes (9). Therefore, ER stress can either serve as a survival means to maintain cell survival, or an important mechanism inducing cell apoptosis (27). In this study, we found that the inhibition of ER stress inhibited the anticancer effects of anacardic acid on apoptosis and autophagy of prostatic cancer.

PI3K/Akt signal pathway transmits multiple growth factor and cytokine signals into cells, influences cell proliferation, survival and apoptosis, and promotes malignant transformation of cells (28). Furthermore, it is related to numerous links such as tumor cell migration, adhesion, tumor angiogenesis and extracellular matrix degradation (13). Abnormally elevated protein expression and kinase activity of PI3K/Akt signal pathway can be seen in multiple human malignant tumors, such as ovarian, prostate, multiple myeloma, breast, pancreatic, lung, endometrial, follicular thyroid cancer and melanoma (13). In addition, the signals that it transmits are remarkably amplified. PI3K/Akt signal pathway is in a central position among numerous signal transduction pathways, and plays an important role in tumor genesis and development (29). However, in the present study, anacardic acid suppressed Akt signaling pathway in prostatic cancer. Xiu et al (30) demonstrated that anacardic acid enhances the proliferation of human ovarian cancer cells through PI3K, VEGF and caspase-3 pathways.
DAPK3 is extensively, but conservatively distributed in multiple species (31). Human DAPK3 locates in chromosome 19, which locates in the cell nucleus as it has nuclear localization signal. DAPK3 forms a dimer through C-terminal domain, thus activating its kinase activity; besides, it induces apoptosis through the phosphorylation of substrates (10). Plenty of data have demonstrated that DAPK3 can exert its apoptosis-inducing function through phosphorylating multiple substrates. In addition, endonuclear DAPK3 can interact with PML body, thus, inducing apoptosis through activating PAR-4 protein by phosphorylation (32). We also detected that the promotion of DAPK3 inhibited the anticancer effects of anacardic acid on autophagy of prostatic cancer.

In summary, we demonstrated that the anacardic acid induces cell apoptosis of prostatic cancer through autophagy by ER stress/DAPK3/Akt signaling pathway (Fig. 15). Therefore, we suggest that anacardic acid shows potential as a possible new drug for therapy of prostatic cancer.

\section{Acknowledgements}

This study was funded by the Inherited Project of Xiangya Famous Doctor of the Third Xiangya Hospital of Central South University.

\section{References}

1. Aggarwal RR, Beer TM, Weinberg VK, Higano C, Taplin ME, Ryan CJ, Lin AM, Alumkal J, Graff JN, Nordquist LT, et al: Intermittent chemotherapy as a platform for testing novel agents in patients with metastatic castration-resistant prostate cancer: A Department of Defense Prostate cancer clinical trials consortium randomized phase II trial of intermittent docetaxel with prednisone with or without maintenance GM-CSF. Clin Genitourin Cancer 13: e191-e198, 2015.

2. Figg WD, Chau CH, Madan RA, Gulley JL, Gao R, Sissung TM, Spencer S, Beatson M, Aragon-Ching J, Steinberg SM, et al: Phase II study of satraplatin and prednisone in patients with metastatic castration-resistant prostate cancer: A pharmacogenetic assessment of outcome and toxicity. Clin Genitourin Cancer 11: 229-237, 2013.

3. Scheltema MJ, van den Bos W, de Bruin DM, Wijkstra H, Laguna MP, de Reijke TM and de la Rosette JJ: Focal vs extended ablation in localized prostate cancer with irreversible electroporation; a multi-center randomized controlled trial. BMC Cancer 16: 299, 2016.

4. Guo J, Huang $\mathrm{X}$, Wang $\mathrm{H}$ and Yang $\mathrm{H}$ : Celastrol induces autophagy by targeting $\mathrm{AR} / \mathrm{miR}-101$ in prostate cancer cells. PLoS One 10: e0140745, 2015.

5. Guo J, Mei Y, Li K, Huang X and Yang H: Downregulation of miR-17-92a cluster promotes autophagy induction in response to celastrol treatment in prostate cancer cells. Biochem Biophys Res Commun 478: 804-810, 2016.

6. Liao H, Xiao Y, Hu Y, Xiao Y, Yin Z and Liu L: microRNA-32 induces radioresistance by targeting DAB2IP and regulating autophagy in prostate cancer cells. Oncol Lett 10: 2055-2062, 2015.

7. Rah B, ur Rasool R, Nayak D, Yousuf SK, Mukherjee D, Kumar LD and Goswami A: PAWR-mediated suppression of BCL2 promotes switching of 3 -azido withaferin A (3-AWA)induced autophagy to apoptosis in prostate cancer cells. Autophagy 11: 314-331, 2015.

8. Mathur A, Abd Elmageed ZY, Liu X, Kostochka ML, Zhang H, Abdel-Mageed AB and Mondal D: Subverting ER-stress towards apoptosis by nelfinavir and curcumin coexposure augments docetaxel efficacy in castration resistant prostate cancer cells. PLoS One 9: e103109, 2014.

9. Yang J, Wei J, Wu Y, Wang Z, Guo Y, Lee P and Li X: Metformin induces ER stress-dependent apoptosis through miR-708-5p/ NNAT pathway in prostate cancer. Oncogenesis 4: e158, 2015. 
10. Fujiwara N, Usui T, Ohama T and Sato K: Regulation of Beclin 1 protein phosphorylation and autophagy by protein phosphatase 2A (PP2A) and death-associated protein kinase 3 (DAPK3). J Biol Chem 291: 10858-10866, 2016.

11. Das TP, Suman S, Papu John AM, Pal D, Edwards A, Alatassi H, Ankem MK and Damodaran C: Activation of AKT negatively regulates the pro-apoptotic function of death-associated protein kinase 3 (DAPK3) in prostate cancer. Cancer Lett 377: 134-139, 2016.

12. Utermark T, Schmit F, Lee SH, Gao X, Schaffhausen BS and Roberts TM: The phosphatidylinositol 3-kinase (PI3K) isoform dependence of tumor formation is determined by the genetic mode of PI3K pathway activation rather than by tissue type. J Virol 88: 10673-10679, 2014.

13. Quan Y, Wang N, Chen Q, Xu J, Cheng W, Di M, Xia W and Gao WQ: SIRT3 inhibits prostate cancer by destabilizing oncoprotein $\mathrm{c}-\mathrm{MYC}$ through regulation of the PI3K/Akt pathway. Oncotarget 6: 26494-26507, 2015.

14. Nambiar J, Bose C, Venugopal M, Banerji A, Patel TB, Kumar GB and Nair BG: Anacardic acid inhibits gelatinases through the regulation of Spry2, MMP-14, EMMPRIN and RECK. Exp Cell Res 349: 139-151, 2016.

15. Philip JY, Da Cruz Francisco J, Dey ES, Buchweishaija J, Mkayula LL and Ye L: Isolation of anacardic acid from natural cashew nut shell liquid (CNSL) using supercritical carbon dioxide. J Agric Food Chem 56: 9350-9354, 2008.

16. Peng C, Zhu J, Sun HC, Huang XP, Zhao WA, Zheng M, Liu LJ and Tian J: Inhibition of histone H3K9 acetylation by anacardic acid can correct the over-expression of Gata4 in the hearts of fetal mice exposed to alcohol during pregnancy. PLoS One 9: e104135, 2014.

17. Alam-Escamilla D, Estrada-Muñiz E, Solís-Villegas E, Elizondo G and Vega L: Genotoxic and cytostatic effects of 6-pentadecyl salicylic anacardic acid in transformed cell lines and peripheral blood mononuclear cells. Mutat Res Genet Toxicol Environ Mutagen 777: 43-53, 2015.

18. Seong YA, Shin PG, Yoon JS, Yadunandam AK and Kim GD Induction of the endoplasmic reticulum stress and autophagy in human lung carcinoma A549 cells by anacardic acid. Cell Biochem Biophys 68: 369-377, 2014

19. Gladwish A, Loblaw A, Cheung P, Morton G, Chung H, Deabreu A, Pang G and Mamedov A: Accelerated hypofractioned postoperative radiotherapy for prostate cancer: A prospective phase I/II study. Clin Oncol (R Coll Radiol) 27: 145-152, 2015.

20. Gong P, Zhang T, He D and Hsieh JT: MicroRNA-145 modulates tumor sensitivity to radiation in prostate cancer. Radiat Res 184: 630-638, 2015

21. Yao K, Jiang X, He L, Tang Y, Yin G, Zeng Q, Jiang Z and Tan J: Anacardic acid sensitizes prostate cancer cells to radiation therapy by regulating H2AX expression. Int J Clin Exp Pathol 8: 15926-15932, 2015.
22. Morell C, Bort A, Vara-Ciruelos D, Ramos-Torres Á, Altamirano-Dimas M, Díaz-Laviada I and Rodríguez-Henche N: Up-regulated expression of LAMP2 and autophagy activity during neuroendocrine differentiation of prostate cancer $\mathrm{LNCaP}$ cells. PLoS One 11: e0162977, 2016.

23. Ramos-Torres Á, Bort A, Morell C, Rodríguez-Henche N and Díaz-Laviada I: The pepper's natural ingredient capsaicin induces autophagy blockage in prostate cancer cells. Oncotarget 7: $1569-1583,2016$

24. Tai S, Xu L, Xu M, Zhang L, Zhang Y, Zhang K, Zhang L and Liang C: Combination of Arsenic trioxide and Everolimus (Rad001) synergistically induces both autophagy and apoptosis in prostate cancer cells. Oncotarget 8: 11206-11218, 2017.

25. Wang L, Fu P, Zhao Y, Wang G, Yu R, Wang X, Tang Z Imperato-McGinley J and Zhu YS: Dissociation of NSC606985 induces atypical ER-stress and cell death in prostate cancer cells. Int J Oncol 49: 529-538, 2016.

26. Bruchmann A, Roller C, WaltherTV, Schäfer G, Lehmusvaara S, Visakorpi T, Klocker H, Cato AC and Maddalo D: Bcl-2 associated athanogene 5 (Bag5) is overexpressed in prostate cancer and inhibits ER-stress induced apoptosis. BMC Cancer 13: 96 , 2013.

27. QiNan W, XiaGuang G, XiaoTian L, WuQuan D, Ling Z and Bing C: Par-4/NF-kappaB mediates the apoptosis of islet beta cells induced by glucolipotoxicity. J Diabetes Res 4692478: 2016, 2016.

28. Qi W, Morales C, Cooke LS, Johnson B, Somer B and Mahadevan D: Reciprocal feedback inhibition of the androgen receptor and $\mathrm{PI} 3 \mathrm{~K}$ as a novel therapy for castrate-sensitive and -resistant prostate cancer. Oncotarget 6: 41976-41987, 2015.

29. Casar B, Rimann I, Kato H, Shattil SJ, Quigley JP and Deryugina EI: In vivo cleaved CDCP1 promotes early tumor dissemination via complexing with activated $\beta 1$ integrin and induction of FAK/PI3K/Akt motility signaling. Oncogene 33: 255-268, 2014.

30. Xiu YL, Zhao Y, Gou WF, Chen S, Takano Y and Zheng HC: Anacardic acid enhances the proliferation of human ovarian cancer cells. PLoS One 9: e99361, 2014.

31. Kwon T, Youn H, Son B, Kim D, Seong KM, Park S, Kim W and Youn B: DANGER is involved in high glucose-induced radioresistance through inhibiting DAPK-mediated anoikis in non-small cell lung cancer. Oncotarget 7: 7193-7206, 2016.

32. Dai L, Ma C, Zhang Z, Zeng S, Liu A, Tang S, Ren Q, Sun Y and $\mathrm{Xu}$ C: DAPK promoter methylation and bladder cancer risk: A systematic review and meta-analysis. PLoS One 11: e0167228, 2016. 